\title{
O papel da autoeficácia parental na saúde mental de mães de crianças com Síndrome da Zika Congênita
}

\author{
The role of parental self-efficacy in the mental health \\ of mothers of children with Congenital Zika Syndrome
}

Tiago Jessé Souza de Lima (https://orcid.org/0000-0001-8840-4285) ${ }^{1}$

Luana Elayne Cunha de Souza (https://orcid.org/0000-0001-9425-9598) ${ }^{2}$

${ }^{1}$ Universidade de Brasília. Campus da UnB, Área Universitária. 73345-010 Brasília DF Brasil. tiago. souzalima@hotmail.com

${ }^{2}$ Universidade de Fortaleza. Fortaleza CE Brasil

\begin{abstract}
The article aimed to evaluate the mediating role of parental self-efficacy (PSE) between socioenvironmental factors and the mental health of the mothers of children with congenital zika syndrome (CZS). The socio-environmental factors were based on the cognitive and affective evaluation that people make of their context (subjective well-being, SWB) and fatigue. Sixty-six mothers with a mean age of 26.4 years of children with CZS in the state of Ceará, participated in this study. A questionnaire with the following scales was applied: Positive and Negative Affect Scale, Life Satisfaction Scale, Fatigue Evaluation Scale, General Health Questionnaire, Parental Self-efficacy Scale. The results indicated that the components of $S W B$, negative affects and life satisfaction, significantly predicted the mental health of mothers of children with CZS, and the PSE predicts better levels of mental health. It was observed that the PSE plays a mediating role in the relationship of satisfaction with life, negative effects and fatigue with mental health. PSE is an important psychological mechanism that acts as a mediator between the socio-environmental context and mental health. Thus, intervention strategies aimed at increasing the feelings of PSE in mothers can have a positive impact on the improvement of their mental health.
\end{abstract}

Key words Zika virus, Mental health, Self-effcacy
Resumo $O$ artigo buscou avaliar o papel mediador da autoeficácia parental (AEP) entre os fatores socioambientais e a saúde mental das mães de crianças com sindrome da Zika congênita (SZC). Os fatores socioambientais foram operacionalizados a partir da avaliação cognitiva e afetiva que as pessoas fazem do seu contexto (Bem-estar subjetivo, BES) e da fadiga. Participaram deste estudo 69 mães de crianças com SZC, com média de idade de 26,4 anos, residentes no estado do Ceará. Foi aplicado um questionário contendo as seguintes escalas: Escala de Afetos Positivos e Negativos, Escala de Satisfação com a Vida, Escala de Avaliação da Fadiga, Questionário de Saúde Geral, Escala de Autoeficácia Parental. Os resultados apontaram que os componentes do BES, afetos negativos e satisfação com a vida, predisseram de forma significativa a saúde mental de mães de crianças com SZC, ademais a AEP prediz melhores niveis de saúde mental. Foi observado que a AEP desempenha um papel mediador na relação da satisfação com a vida, dos afetos negativos e da fadiga com a saúde mental. A AEP é um importante mecanismo psicológico que atua como mediador entre o seu contexto socioambiental e sua saúde mental. Dessa forma, estratégias de intervenções voltadas para aumentar os sentimentos de AEP em mães poderão ter um impacto positivo na melhora da sua saúde mental.

Palavras-chave Zika vírus, Saúde mental, Autoeficácia 


\section{Introdução}

Os relatos iniciais de 2015 sobre a relação entre o Zika vírus e a microcefalia levaram a um esforço internacional de pesquisas para compreensão dos condicionantes relacionados com a transmissão do Zika vírus e sua relação com defeitos de nascença, tais como a microcefalia, deficiência intelectual, paralisia cerebral, epilepsia, dificuldade de deglutição, anomalias dos sistemas visual e auditivo, além de distúrbio do comportamento ${ }^{1-3}$. Esse padrão distinto de defeitos congênitos, que podem estar associados a outras anomalias, foi denominado de Síndrome Congênita do Zika vírus $(\mathrm{SCZ})^{4}$.

Assim como ocorre com outras condições crônicas, a exemplo da paralisia cerebral ${ }^{5}$, transtorno do espectro autista ${ }^{6}$, síndrome de Down e síndrome de Rett ${ }^{7}$, o cuidado com um bebê com SCZ apresenta diversas implicações psicossociais para a dinâmica da família, sobretudo para as mães, que estão mais envolvidas nos cuidados diários com esse bebêe. As pesquisas realizadas com mães de crianças com condições crônicas têm apontado, de forma consistente, que elas apresentam menor qualidade de vida e níveis mais elevados de fadiga, estresse, ansiedade e depressã $0^{7,9,10}$, comparativamente às mães de crianças com desenvolvimento típico. Os estudos indicando os impactos na saúde mental de mães de crianças com SCZ, apesar de escassos, apontam um quadro similar ao observado com outras condições crônicas, apontando níveis elevados de ansiedade, fadiga, menor qualidade de vida e pior saúde mental de forma global ${ }^{11,12}$.

Uma criança com condições crônicas tem múltiplas necessidades de cuidado que vão além das de uma criança da mesma idade com desenvolvimento típico, consequentemente, exige um maior envolvimento dos pais no seu cuidado? De acordo com Souza et al. ${ }^{12}$ as mães e pais dessas crianças precisam, por exemplo, lidar com a aceitação das condições e limitações dos filhos, se ajustando e gerenciando as dificuldades desses, podem enfrentar sofrimento emocional significativo, conflitos no relacionamento, problemas financeiros, estigmatização, isolamento social e atitudes sociais negativas, além do tempo consumido nas consultas a diversos especialistas ${ }^{13,14}$. Ademais, Bailey e Ventura ${ }^{2}$, apontam que, as famílias de crianças com SZC podem estar expostas a condições ainda mais críticas, sobretudo devido às incertezas das consequências da SZC, ainda pouco estudadas na literatura médica, para a vida futura da criança.
Mediante essa realidade, o envolvimento ativo das mães nos cuidados com as crianças com SZC é extremamente necessário para que o tratamento da síndrome e de suas consequências seja efetivo. Um dos fatores que influenciam esse envolvimento materno é a autoeficácia parental (AEP). A autoeficácia compreende as crenças que os indivíduos têm sobre sua capacidade de organizar e desempenhar condutas necessárias para alcançar suas metas e objetivos ${ }^{15}$. A AEP se refere às crenças que os pais têm sobre suas habilidades de prover cuidados aos filhos de forma bem-sucedida ${ }^{16}$. Bandura ${ }^{15}$ aponta que a autoeficácia afeta diretamente o comportamento, já que pessoas com maior autoeficácia têm maior probabilidade de persistir mediante tarefas difíceis.

Diversos estudos têm apontado que uma maior AEP está associada ao uso de estratégias parentais positivas, enfrentamento e persistência na maternagem ${ }^{17}$. Ademais, os pais com maior AEP relatada são mais propensos a se envolver no tratamento dos seus filhos, levando a maiores níveis de melhora durante o tratamento ${ }^{18}$. Por outro lado, uma baixa AEP tem se mostrado relacionada com a depressão materna ${ }^{19}$, com o estresse parental, padrões disfuncionais de interação familiar, problemas de saúde física e mental dos pais, excitação emocional parental negativa e diminuição da qualidade das interações entre pais e filhos ${ }^{20}$. Pais que possuem baixa AEP tendem a fazer mais atribuições internas de fracasso e manifestam graus mais altos de ansiedade e sintomas depressivos ${ }^{21}$.

Em geral, a avaliação dos pais sobre sua capacidade de realizar com eficácia as tarefas parentais tem consequências importantes para práticas parentais consistentes e para o desenvolvimento infantil ${ }^{20}$. De acordo com o modelo de Bandura, os pais que possuem um alto senso de AEP acreditam que eles têm as habilidades e qualidades necessárias para ter uma influência positiva no comportamento e desenvolvimento de seus filhos e tendem a persistir em situações difíceis para os pais. Pais com baixa autoeficácia parental correm o risco de experimentar ansiedade, depressão, estresse e ter resultados mais desfavoráveis no tratamento de seus filhos.

As condições socioambientais têm se mostrado como importantes preditores da $\mathrm{AEP}^{16}$. De acordo com a teoria de Bandura ${ }^{15}$, as crenças de autoeficácia são desenvolvidas e moldadas por experiências de sucesso e fracasso, dificuldade percebida de tarefas, interações interpessoais com outros e excitação fisiológica e psicológica aversiva (por exemplo, ansiedade). A AEP pode 
funcionar como um mediador entre essas variáveis socioambientais e a saúde mental dos pais, ou seja, a avaliação que os pais fazem dessas condições podem minar a sua AEP e explicar comportamentos parentais menos adaptativos e, consequente, pior saúde mental ${ }^{16,17}$.

O papel mediador da AEP, entre variáveis socioambientais e a saúde mental dos pais (estresse, ansiedade e depressão), tem sido observado em diversos estudos na literatura com amostras de pais de crianças com autismo ${ }^{22-24}$. No entanto, não foi encontrado nenhum estudo que testasse o papel mediador da AEP entre os fatores socioambientais e a saúde mental das mães de crianças com SZC e, portanto, essa é a lacuna que o presente estudo procura preencher.

No presente estudo, os fatores socioambientais são operacionalizados a partir da avaliação cognitiva que as pessoas fazem do seu contexto social, assim como dos sentimentos positivos e negativos advindos desse. Tal avaliação, tem sido denominada na literatura de bem-estar subjetivo $(B E S)^{25}$, que engloba a satisfação geral com a vida (BES cognitivo) e os afetos positivos e negativos (BES afetivo). A satisfação com a vida refere-se a uma avaliação cognitiva da vida como um todo, já os afetos positivos expressam a frequência de emoções positivas experimentadas pelo indivíduo e os afetos negativos a frequência de emoções negativas ${ }^{26}$.

Ademais, o desgaste diário provocado pelo contexto socioambiental pode ser um importante fator associado à AEP e à saúde mental. Essa experiência de desgaste diário tem sido trabalhada na literatura através do conceito de fadiga, definida como um sentimento duradouro de exaustão física e mental que não é necessariamente causado por exercícios e que não é facilmente aliviado pelo repouso ${ }^{17}$. De acordo com Yang e $\mathrm{Wu}^{27}$, a fadiga não é necessariamente um sintoma de uma determinada doença, mas pode ser entendida como uma experiência diária de desgaste, que pode ter um impacto negativo nas funções cognitivas, tais como concentração, memória e tomada de decisão, levando também a uma menor disposição para desenvolver ou manter objetivos ${ }^{28}$.

Nesse sentido, o presente artigo teve por objetivo geral testar o papel mediador da AEP entre o BES (satisfação com a vida, afetos positivos e negativos), a fadiga e a saúde mental das mães de crianças com SZC. De maneira específica, inicialmente será avaliada a relação do BES e da fadiga com a saúde mental de mães de crianças com SZC. Em seguida, para as variáveis que predisserem significativamente a saúde mental, será avaliado se essa relação é mediada pela AEP reportado por essas mães. O modelo a ser testado é apresentado na Figura 1.

\section{Método}

\section{Participantes}

Participaram deste estudo 69 mães de crianças com SZC, com média de idade de 26,4 anos $(\mathrm{DP}=6,23)$, variando entre 16 e 42 anos, sendo a maioria autodeclarada parda $(49,3 \%)$, com $37,5 \%$ tendo informado ter cursado o ensino fundamental completo e $39,3 \%$ completado o ensino médio. Em relação à inserção no mercado de trabalho, $64,8 \%$ indicou não trabalhar, sendo que a maioria $(71,9 \%)$ indicou ter renda familiar de até 1 salário mínimo. Aproximadamente $25 \%$ da amostra reside na região Metropolitana de Fortaleza enquanto a outra parte é composta por residentes de diferentes cidades do Estado do Ceará.

\section{Instrumento}

O Bem-estar subjetivo foi avaliado através da (1) Escala de Afetos Positivos e Negativos (PANAS) ${ }^{29}$ e da Escala de Satisfação com a Vida $(\mathrm{SWLS})^{30}$. A PANAS é composta por dez adjetivos (cinco positivos e cinco negativos) que avaliam o quanto o participante tem experimentado cada uma destes afetos nos últimos dias, sendo respondidos uma escala de resposta de sete pontos $(1=$ Nada a $7=$ Extremamente $)$. Os fatores afetos positivos $(\alpha=0,83)$ e afetos negativos $(\alpha=0,72)$ obtiveram índices adequados de consistência interna (alfa de Cronbach). A SWLS é composta por cinco itens que avaliam a satisfação com aspectos gerais da vida. As respostas são dadas de acordo com uma escala de 7 pontos $(1=$ Discordo totalmente a 7 = Concordo totalmente), tendo apresentado uma consistência interna igual a 0,72.

A fadiga foi mensurada através da Escala de Avaliação da Fadiga ${ }^{28}$. Composta por 10 itens respondidos em escala de cinco pontos ( $1=$ Nunca a $5=$ Sempre), devendo o participante indicar como tem se sentido, de forma geral, no seu dia a dia. A escala apresentou boa consistência interna $(\alpha=0,78)$.

A autoeficácia parental foi mensurada através de oito itens da Revised Caregiving Appraisal Scales, subescala Caregiving mastery ${ }^{31}$ adaptados para a realidade de cuidados das mães de crianças com SZC. As mães eram solicitadas a indicarem 


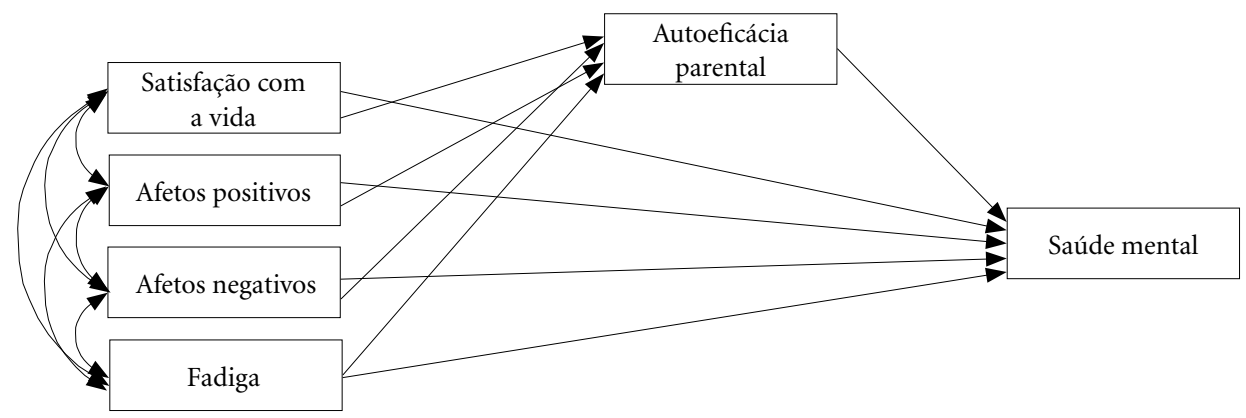

Figura 1. Modelo proposto de mediação da autoeficácia parental.

sua concordância com afirmações relacionadas com os cuidados dos filhos com SZC, utilizando uma escala de resposta variando de $1=$ Discordo muito a 5 = Concordo muito. Essa escala apresentou um alfa de Cronbach de 0,79.

A saúde mental foi mensurada através do Questionário de Saúde Geral (QSG-12) 32,33. Composta por 12 itens, sendo utilizada para avaliar sintomas psiquiátricos não psicóticos. Essa medida é aqui utilizada para avaliar a saúde mental dos pais. No caso de itens negativos as alternativas de resposta variam de $1=$ Absolutamente não, a 4 = Muito mais que de costume; em caso de itens positivos as respostas variaram de $1=$ Mais que de costume, a $4=$ Muito menos que de costume. Uma maior pontuação é indicativa de um menor nível de saúde mental. $O$ índice de consistência interna da escala também é adequado $(\alpha=0,80)$.

Os participantes também responderam uma série de perguntas de caráter sociodemográfico (idade, escolaridade, situação de emprego, renda familiar, número de filhos).

\section{Procedimento}

Esta pesquisa foi realizada durante um mutirão de atendimento a crianças diagnosticados com SZC do Estado do Ceará. Trata-se de um evento organizado por uma equipe multiprofissional que tem como objetivo dar atendimento e fazer um acompanhamento periódico do desenvolvimento dessas crianças. Esta pesquisa faz parte de um projeto maior que conta com aprovação do comitê de ética em pesquisa do Hospi- tal Infantil Albert Sabin-CE. As mães eram abordadas individualmente por algum dos membros da equipe, nesse momento inicial o objetivo da pesquisa era explicado, assim como era salientado o caráter voluntário e anônimo das respostas. Aquelas que concordaram em participar da pesquisa assinaram o termo de consentimento livre e esclarecido e, em seguida, foram entrevistadas. Em média, 20 minutos foram suficientes para concluir cada participação.

\section{Análise dos dados}

Inicialmente, foram computadas correlações de Pearson para avaliar as possíveis correlações entre a saúde mental e as demais variáveis estudadas. As variáveis que apresentaram correlação significativa nessa etapa foram incluídas posteriormente em uma regressão múltipla, hierárquica, com método Enter. Na primeira equação incluiu-se o bloco das variáveis sociodemográficas. Na segunda equação acrescentou-se as variáveis psicossociais. Posteriormente, para testar o papel mediador da autoeficácia, foi utilizada a macro PROCESS desenvolvida para o SPSS por Hayes e Rockwood ${ }^{34}$. Para avaliar a significância da mediação, especificamente a confiabilidade dos efeitos indiretos, foi utilizado o bootstrapping. $\mathrm{O}$ bootstrapping é um método computacional que envolve repetidas amostragens extraídas do conjunto de dados, estimando o efeito indireto em cada uma delas. A hipótese da mediação é aceita se os intervalos de confiança obtidos através do bootstrapping não contenham o valor zero, 
ou seja, o efeito indireto teria um valor diferente de zero ${ }^{35}$. Foram computadas 1.000 amostras de bootstrapping e o intervalo de confiança adotado foi de $90 \%$, sendo calculados através do método bias corrected and accelerated (BCa).

\section{Resultados}

Inicialmente, foram realizadas análises de correlação entre a saúde mental e as variáveis sociodemográficas e psicossociais (Tabela 1). Os resultados indicaram que, das variáveis sociodemográficas, a variável número de filhos foi a única a apresentar uma correlação significativa, sendo que ao aumentar o número de filhos o nível de saúde mental diminui. No entanto, cabe salientar que essa foi uma correlação considerada como fraca $^{36}$. Entre as variáveis psicossociais, os afetos positivos, a satisfação com a vida e a autoeficácia estão relacionados com melhores níveis de saúde mental, enquanto que os afetos negativos e a fadiga se relacionam com piores níveis de saúde mental. As correlações entre as variáveis psicossociais com a saúde mental podem ser consideradas como moderadas ${ }^{36}$. Embora esse estudo não objetive uma análise do efeito das variáveis sociodemográficas na saúde mental das mães de crianças com SZC, elas foram introduzidas nas análises com o objetivo de controlarmos seus efeitos, o que possibilita uma análise mais precisa dos efeitos das demais variáveis preditivas.

Na sequência, foi realizada uma análise de regressão hierárquica (Tabela 2). No primeiro Modelo, a saúde mental foi regredida na variável sociodemográfica Número de filhos, obtendo-se um coeficiente de regressão significativo, $R^{2}=0,08, F$ $(1,67)=5,65, p<0,05$. A variável em questão prediz significativamente a saúde mental, de modo que, ao aumentar o número de filhos o nível de saúde mental diminui. No segundo passo, quando as variáveis do BES e a fadiga são acrescentadas ao modelo anterior, o coeficiente de regressão se mantém significativo, $R^{2}=0,53, F(5,62)=13,9$, $p<0,001$. Observa-se que as variáveis afetos negativos e fadiga predizem piores níveis de saúde mental, enquanto a variável satisfação com a vida prediz melhores níveis de saúde mental. As variáveis número de filhos e afetos positivos não foram significativas. Esse segundo modelo apresentou um incremento significativo no percentual explicado da variância da saúde mental das mães: $R_{\text {Change }}^{2}=0,49 ; F_{\text {Change }}(4,62)=14,7, p<0,001$.

Em seguida, foram realizadas análises de regressão simples para testar a hipótese de que
Tabela 1. Correlações entre saúde mental e variáveis sociodemográficas e psicossociais.

\begin{tabular}{lc}
\hline & Saúde mental \\
\hline Idade & 0,14 \\
Escolaridade & $-0,01$ \\
Situação de emprego & 0,18 \\
Renda familiar & $-0,05$ \\
Número de filhos & $0,28^{\star}$ \\
Afetos positivos & $-0,36^{* \star}$ \\
Afetos negativos & $0,60^{* *}$ \\
Satisfação com a vida & $-0,55^{\star *}$ \\
Fadiga & $0,64^{* \star}$ \\
Autoeficácia & $-0,49^{* *}$ \\
\hline
\end{tabular}

Nota: Situação de emprego: $0=$ desempregado; $1=$ empregado. ${ }^{*} \mathrm{p}<0,05 ;{ }^{* *} \mathrm{p}<0,01$.

a autoeficácia atua como mediador na relação entre as variáveis psicossociais e a saúde mental. Seguindo as análises anteriores, as mediações foram testadas apenas para as variáveis que predisseram significativamente a saúde mental. Os resultados das análises de regressão com os pesos de regressão padronizados são apresentados na Figura 2. Podemos observar que a autoeficácia responde parcialmente pelo efeito dos preditores na saúde mental, de modo que o efeito total de cada preditor na saúde mental (caminho d) foi reduzido ao se acrescentar a autoeficácia na regressão (caminho c, efeito direto), embora se tenham mantido significativos. Essa redução no efeito, calculada pela subtração entre os pesos de regressão dos caminhos $d$ e $c$, é denominada de efeito indireto (Tabela 3). Os efeitos indiretos obtidos foram submetidos a uma análise de bootstrapping para geração de intervalos de confiança. Observa-se, na Tabela 3, que os intervalos obtidos não contêm o valor zero, o que indica que o efeito indireto da autoeficácia é significativamente diferente de zero. Portanto, a autoeficácia atua como mediador entre as variáveis preditoras (afetos negativos, satisfação com a vida e fadiga) e a saúde mental.

\section{Discussão}

O presente artigo teve por objetivo geral testar o papel mediador da AEP entre os fatores socioambientais e a saúde mental das mães de crianças com SZC. Para tanto, foi inicialmente avaliada a relação do BES e da fadiga com a saúde mental de 
Tabela 2. Análise de regressão hierárquica dos preditores da saúde mental.

\begin{tabular}{|c|c|c|c|c|c|c|c|}
\hline \multirow{2}{*}{ Preditores } & \multicolumn{3}{|c|}{ Passo 1} & \multicolumn{4}{|c|}{ Passo 2} \\
\hline & b & IC95\% & $\beta$ & b & IC95\% & $\boldsymbol{\beta}$ & $\eta_{p}^{2}$ \\
\hline Constante & 2,01 & {$[1,84 ; 2,19]$} & & 1,66 & {$[0,89 ; 2,44]$} & & \\
\hline Número de filhos & 0,14 & {$[0,02 ; 0,25]$} & $0,28^{*}$ & 0,06 & {$[-0,03 ; 0,15]$} & 0,13 & 0,03 \\
\hline Afetos positivos & & & & $-0,01$ & {$[-0,10 ; 0,07]$} & $-0,03$ & 0,00 \\
\hline Afetos negativos & & & & 0,11 & {$[0,01 ; 0,23]$} & $0,24^{\star}$ & 0,06 \\
\hline Satis. com a vida & & & & $-0,12$ & {$[-0,22 ;-0,01]$} & $-0,25^{\star}$ & 0,08 \\
\hline Fadiga & & & & 0,25 & {$[0,07 ; 0,43]$} & $0,34^{* *}$ & 0,11 \\
\hline $\mathrm{R}^{2}$ & & 0,08 & & & & & 0,53 \\
\hline$R_{\text {Ajustado }}^{2}$ & & 0,07 & & & & & 0,49 \\
\hline
\end{tabular}

Nota: ${ }^{*} \mathrm{p}<.05 ;{ }^{* *} \mathrm{p}<.01$.

Tabela 3. Coeficientes de regressão para o teste do efeito mediador da Autoeficácia Parental.

\begin{tabular}{lccc}
\hline \multirow{2}{*}{ Preditores } & \multirow{2}{*}{ Efeito indireto } & \multicolumn{2}{c}{ Boostraping [IC95\%] } \\
\cline { 3 - 4 } & & Inferior & Superior \\
\hline Afetos negativos & 0,04 & 0,001 & 0,097 \\
Satisfação com a vida & $-0,04$ & $-0,109$ & $-0,005$ \\
Fadiga & 0,06 & 0,006 & 0,146 \\
\hline
\end{tabular}
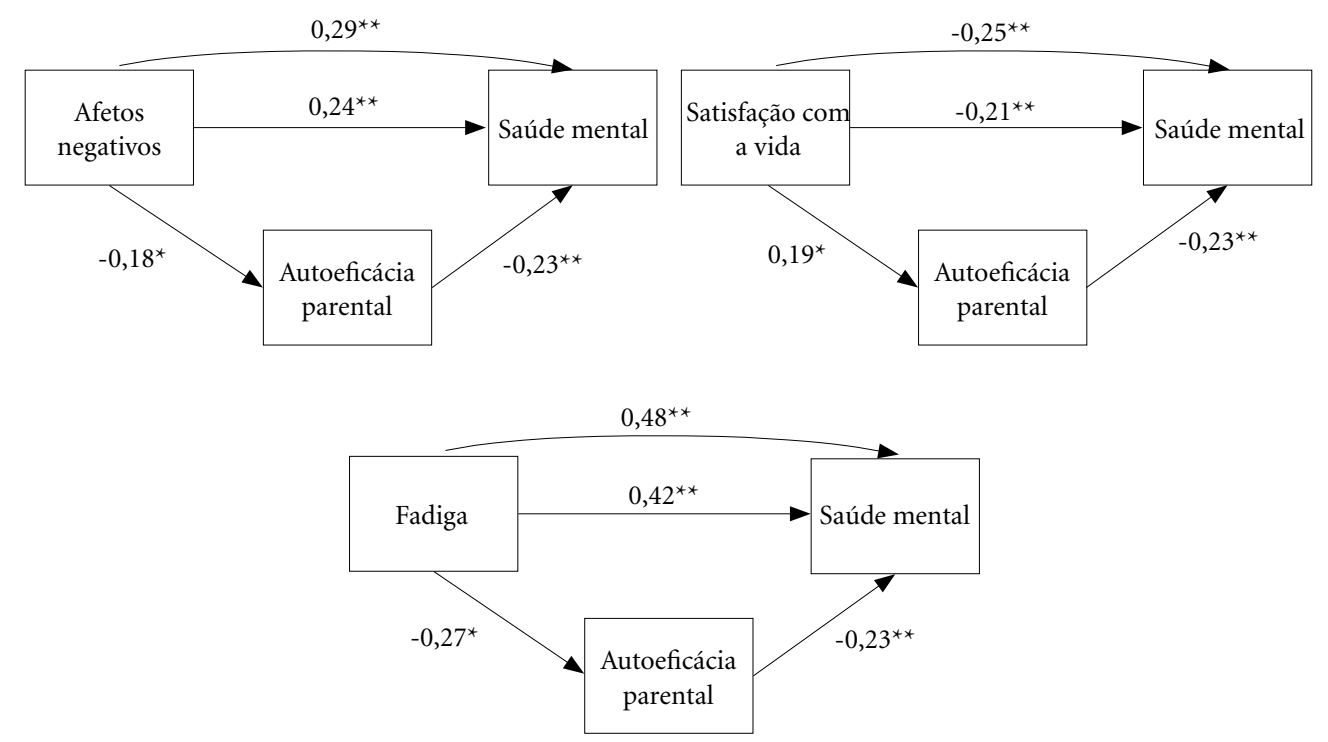

Figura 2. Análises de regressão dos efeitos mediadores da Autoeficácia Parental. 
mães de crianças com SZC, e posteriormente se essa relação é mediada pelo nível de autoeficácia parental reportado por essas mães. Os resultados obtidos forneceram evidências sobre a relação preditiva do BES e da Fadiga na saúde mental, assim como apontaram que a AEP mediou essa relação.

Os componentes do BES, afetos negativos e satisfação com a vida, predisseram de forma significativa a saúde mental de mães de crianças com SZC, corroborando assim outros estudos na literatura, conduzidos com mães e pais de crianças com condições crônicas, que indicam uma relação entre a satisfação com a vida e os afetos negativos com a saúde mental ${ }^{37-39}$. Nesse mesmo sentido, a fadiga também apresentou um efeito negativo sobre a saúde mental das mães de crianças com SZC. De fato, diversas pesquisas com mães e pais de crianças com condições crônicas têm apontado que a fadiga é um forte fator de risco para o desenvolvimento de problemas psicológicos ${ }^{17,40}$, sendo esperado que o mesmo padrão seja observado em relação às mães de crianças com SZC. De acordo com Giallo et al. ${ }^{17}$, a fadiga física e mental pode tornar mais difícil para os pais acessar seus recursos de enfrentamento pessoal para gerenciar fatores estressores do ambiente, colocando-os em risco de desenvolver problemas que afetem sua saúde mental, a exemplo da depressão.

Embora a relação da satisfação com a vida, dos afetos negativos e da fadiga com a saúde mental tenha sido bem estabelecida na literatura, os possíveis mecanismos responsáveis por parte dessa relação precisam ser melhor esclarecidos. Uma possível variável que pode explicar esse efeito é AEP. Os resultados apontaram que a AEP funciona como mediador dessa relação, respondendo parcialmente pelos efeitos dos preditores na saúde mental das mães de crianças com SZC. Por ser uma crença na própria capacidade de organizar e executar cursos de ações, a autoeficácia atua como um buffer, ou seja, perceber uma situação como estressante ou ameaçadora depende, em parte, das habilidades de enfrentamento que o indivíduo possui e da crença na sua capacidade de lidar com essa situação ${ }^{41}$.

Nas análises de mediação, podemos observar que a AEP prediz melhores níveis de saúde geral. Estudos anteriores apontam que, de fato, uma baixa AEP está relacionada com a depressão em mães ${ }^{20}$ e com ansiedade nos pais ${ }^{42}$. Embora não possamos inferir causalidade nos resultados observados, teorias sobre distress e autoeficácia ${ }^{20}$ sugerem uma relação bidirecional e interativa em que a ansiedade e a depressão levam a atribuições negativas de AEP. Por sua vez, AEP baixa leva à parentalidade mais precária, como falta de persistência e práticas parentais inconsistentes. Isso leva ao fracasso, angústia e afirmação da percepção da baixa capacidade parental. Podemos observar também que a autoeficácia é predita pelas variáveis do BES, afetos negativos e satisfação com a vida, e pela fadiga. A AEP está relacionada, e pode ser predita, por experiências de sucesso e fracasso relacionadas ao meio no qual os indivíduos estão inseridos ${ }^{15}$, da dificuldade percebida das tarefas com as quais eles têm que lidar, interações interpessoais com outros e excitação fisiológica e psicológica aversiva (afetos negativos, por exemplo). Esses resultados são consistentes com pesquisas anteriores com pais de crianças com condições crônicas, que observaram que a gravidade dos sintomas infantis e, particularmente, o comportamento problemático relacionado a essas condições, correlacionam-se com a autoeficácia parental ${ }^{42,43}$.

Ademais, os resultados apontaram que a AEP desempenha um papel de mediador na relação da satisfação com a vida, dos afetos negativos e da fadiga com a saúde mental. De acordo com Smart $^{20}$, modelos explicativos que incluíram a AEP, variáveis relacionadas com o cuidado e sofrimento dos pais, descobriram que o AEP desempenha um papel importante no caminho entre as variáveis de cuidado e o sofrimento dos pais como um mediador parcial. Embora a AEP possa se relacionar de forma interativa com a saúde mental das mães de crianças com SZC, o efeito da AEP na saúde mental dessas mães também se dá de forma indireta, como um mediador de outras variáveis. Esses resultados são coerentes com outros estudos da literatura ${ }^{20,24,42}$.

Os achados de que a autoeficácia pode atuar como um potencial mediador tem implicações práticas e teóricas. Do ponto de vista teórico, o papel mediador da autoeficácia pode nos levar a compreendê-la como um fator compensatório que tem um efeito positivo na saúde mental da mães independente do nível de dificuldade percebido na maternagem, ou seja, uma elevada AEP parental tem um efeito positivo na saúde mental das mães, tenham elas ou não uma percepção de dificuldade na maternagem. Esse efeito possivelmente ocorre em função do alto nível de envolvimento das mães nos cuidados com seus filhos ${ }^{42}$. É possível que esses resultados não sejam replicados ao se estudar amostras com pais, o que reflete os papéis sociais tradicionalmente desempenhados pelas mulheres, que continuam 
a assumir a responsabilidade primária pela assistência aos filhos e por outros trabalhos de serviço doméstico ${ }^{8}$. No entanto, estudos futuros poderiam buscar replicar esses achados em amostras de mães e pais.

Quanto as suas implicações práticas, os resultados sugerem que intervenções voltadas para aumentar os sentimentos de AEP em mães poderão ter um impacto direto na melhora da sua saúde mental. Em uma metanálise de 10 estudos sobre intervenções focadas no aumento da AEP, Amin, Tam e Shorey ${ }^{44}$ apontam que os resultados são positivos, levando a um aumento da AEP que se mantêm por até 15 semanas após as intervenções. Esses mesmos autores apontam que prover intervenções universais de educação dos pais que enfoquem o aprimoramento da AEP funciona como fator protetivo contra os efeitos prejudiciais da fadiga e da angústia psicológica que os pais podem experimentar ${ }^{44}$.

Embora os resultados obtidos sejam consistentes teoricamente com outros estudos da literatura, algumas limitações podem ser apontadas. Uma primeira limitação diz respeito à amostra que pode não ser representativa do universo de mães de crianças com SZC. Outros estudos deverão contar com amostras mais representativas. Ademais, as mães que participaram desse estudo haviam procurado serviços de saúde especializados para os filhos, onde foram entrevistadas, o que pode estar diretamente vinculado a um maior nível de AEP. Isso pode indicar que essas mães podem apresentar um comportamento mais proativo e de busca de apoio e tratamento para os filhos, fazendo uso de estratégias de enfrentamento mais adaptativas ${ }^{45}$. Entrevistar mães que não se encontram nesses serviços pode trazer um panorama diferente do aqui apresentado, já que essas podem ter níveis mais reduzidos de AEP. O delineamento correlacional aqui adotado também não nos permite inferir relações de causa e efeito e nem assegurarmos a direção dos efeitos entre as variáveis. Novas pesquisas, utilizando desenhos longitudinais e com grupo controle poderão identificar de forma mais precisa a fiabilidade da direção das relações entre as variáveis.

Em síntese, o presente estudo apresenta uma contribuição inédita para a compreensão do papel da AEP na saúde mental de mães de crianças com SZC. A AEP é um importante mecanismo psicológico que atua como mediador entre o seu contexto socioambiental e sua saúde mental. Dessa forma, estratégias de intervenções voltadas para aumentar os sentimentos de AEP em mães poderão ter um impacto positivo na melhora da sua saúde mental.

\section{Colaboradores}

TJS Lima colaborou na elaboração da introdução, análise dos dados e discussão dos resultados. LEC Souza participou na discussão dos resultados e revisão final do manuscrito. 
1. Honein MA, Jamieson DJ. Revealing the Effects of Zika-Detection of Brain Abnormalities and Other Disabilities Associated with Congenital Infection. JAMA Pediatr 2019; 173(1):16.

2. Bailey DB, Ventura LO. The Likely Impact of Congenital Zika Syndrome on Families: Considerations for Family Supports and Services. Pediatrics 2018; 141(Supl. 2):S180-S187.

3. Eickmann SH, Carvalho MDCG, Ramos RCF, Rocha MÂW, van der Linden V, Silva PFS. Síndrome da infecção congênita pelo vírus Zika. Cad Saude Publica 2016; 32(7):1-3.

4. Moore CA, Staples JE, Dobyns WB, Pessoa A, Ventura C V., Da Fonseca EB, Ribeiro EM, Ventura LO, Neto $\mathrm{NN}$, Arena JF, Rasmussen SA. Characterizing the pattern of anomalies in congenital zika syndrome for pediatric clinicians. JAMA Pediatr 2017; 171(3):288-295.

5. Lee MH, Matthews AK, Park C. Determinants of Health-related Quality of Life Among Mothers of Children With Cerebral Palsy. J Pediatr Nurs 2019; 44:1-8.

6. Iadarola S, Pérez-Ramos J, Smith T, Dozier A. Understanding stress in parents of children with autism spectrum disorder: a focus on under-represented families. Int J Dev Disabil 2019; 65(1):20-30.

7. Mori Y, Downs J, Wong K, Leonard H. Longitudinal effects of caregiving on parental well-being: the example of Rett syndrome, a severe neurological disorder. Eur Child Adolesc Psychiatry 2019; 28(4):505-520.

8. Wood W, Eagly AH. Biosocial Construction of Sex Differences and Similarities in Behavior. Advances Experimental Soc Psychol 2012; 46:55-123.

9. Guillamón N, Nieto R, Pousada M, Redolar D, Muñoz E, Hernández E, Boixadós M, Gómez-Zúñiga B. Quality of life and mental health among parents of children with cerebral palsy: The influence of self-efficacy and coping strategies. J Clin Nurs 2013; 22(1112):1579-1590.

10. Gatzoyia D, Kotsis K, Koullourou I, Goulia P, Carvalho AF, Soulis S, Hyphantis T. The association of illness perceptions with depressive symptoms and general psychological distress in parents of an offspring with autism spectrum disorder. Disabil Health J 2014; 7(2):173-180.

11. Santos Oliveira SJG, Melo ES, Reinheimer DM, Gurgel RQ, Santos VS, Martins-Filho PRS. Anxiety, depression, and quality of life in mothers of newborns with microcephaly and presumed congenital Zika virus infection. Arch Womens Ment Health 2016; 19(6):1149-1151.

12. Souza LEC, Lima TJS, Ribeiro EM, Pessoa ALS, Figueiredo TC, Lima LBP. Mental Health of Parents of Children with Congenital Zika Virus Syndrome in Brazil. $J$ Child Fam Stud 2018; 27(4):1207-1215.

13. Mak WWS, Kwok YTY. Internalization of stigma for parents of children with autism spectrum disorder in Hong Kong. Soc Sci Med 2010; 70(12):2045-2051.

14. Pozo P, Sarriá E, Brioso A. Family quality of life and psychological well-being in parents of children with autism spectrum disorders: A double ABCX model. J Intellect Disabil Res 2014; 58(5):442-458.
15. Bandura A. Social cognitive theory: An agentic perspective. Annu Rev Psychol 2001; 52(1):1-26.

16. Jones TL, Prinz RJ. Potential roles of parental self-efficacy in parent and child adjustment: A review. Clin Psychol Rev 2005; 25(3):341-363.

17. Giallo R, Wood CE, Jellett R, Porter R. Fatigue, wellbeing and parental self-efficacy in mothers of children with an Autism Spectrum Disorder. Autism 2013; 17(4):465-480.

18. Warren JS, Brown CR, Layne CM, Nelson PL. Parenting self-efficacy as a predictor of child psychotherapy outcomes in usual care: A multi-dimensional approach. Psychother Res 2011; 21(1):112-123.

19. Meirsschaut M, Roeyers H, Warreyn P. Parenting in families with a child with autism spectrum disorder and a typically developing child: Mothers' experiences and cognitions. Res Autism Spectr Disord 2010; 4(4):661-669.

20. Smart LK. Parenting Self-Efficacy in Parents of Children with Autism Spectrum Disorders [dissertação]. Utah: Universidade Brigham Young; 2016.

21. Miller AC, Gordon RM, Daniele RJ, Diller L. Stress, appraisal, and coping in mothers of disabled and nondisabled children. J Pediatr Psychol 1992; 17(5):587605.

22. Goldberg SEM. Connectedness in Mothers of Children with Autism Spectrum Disorder: Associations with Maternal Stress, Self-Efficacy, and Empathy [tese]. Oakland: Mills College; 2018.

23. Factor RS. Autism spectrum disorder traits and parental stress: The moderating role of parental self-efficacy [tese]. Virginia: Instituto Politécnico e Universidade Estadual da Virgínia; 2016.

24. Rezendes DL, Scarpa A. Associations between Parental Anxiety/Depression and Child Behavior Problems Related to Autism Spectrum Disorders: The Roles of Parenting Stress and Parenting Self-Efficacy. Autism Res Treat 2011; 395190.

25. Diener E, Chan MY. Happy People Live Longer: Subjective Well-Being Contributes to Health and Longevity. Appl Psychol Heal Well-Being 2011; 3(1):1-43.

26. Woyciekoski C, Stenert F, Hutz CS. Determinantes del bienestar subjetivo. Psico (Porto Alegre) 2012; 43(3):280-288.

27. Yang $\mathrm{CM}, \mathrm{Wu} \mathrm{CH}$. The Situational Fatigue Scale: A different approach to measuring fatigue. Qual Life Res 2005; 14(5):1357-1362.

28. Michielsen HJ, De Vries J, Van Heck GL, Van de Vijver FJR, Sijtsma K. Examination of the Dimensionality of Fatigue: The Construction of the Fatigue Assessment Scale (FAS). Eur J Psychol Assess 2004; 20(1):39-48.

29. Watson D, Clark LA, Tellegen A. Development and validation of brief measures of positive and negative affect: The PANAS scales. J Pers Soc Psychol 1988; 54(6):1063-1070.

30. Diener E, Emmons RA, Larsen RJ, Griffin S. The Satisfaction With Life Scale. J Pers Assess 1985; 49(1):71-75.

31. Lawton MP, Moss M, Hoffman C, Perkinson M. Two Transitions in Daughters' Caregiving Careers. Gerontologist 2000; 40(4):437-448. 
32. Goldberg DP, Gater R, Sartorius N, Ustun TB, Piccinelli M, Gureje O, Rutter C. The validity of two versions of the GHQ in the WHO study of mental illness in general health care. Psychol Med 1997; 27(1):191197.

33. Gouveia VV, Lima TJS, Gouveia RSV, Freires LA, Barbosa LHGM. Questionário de Saúde Geral ( QSG-12 ): o efeito de itens negativos em sua estrutura fatorial. Cad Saude Publica 2012; 28(2):375-384.

34. Hayes AF, Rockwood NJ. Regression-based statistical mediation and moderation analysis in clinical research: Observations, recommendations, and implementation. Behav Res Ther 2017; 98:39-57.

35. Souza LAS, Torres ARR, Barbosa GA, Lima TJS, Souza LEC. Subjective well-being and burnout in military cadets: The mediating role of self-efficacy. Psicol Reflex Crit 2015; 28(4):744-752.

36. Schober P, Schwarte LA. Correlation coefficients: Appropriate use and interpretation. Anesth Analg 2018; 126(5):1763-1768.

37. Souza LEC, Lima TJS, Ribeiro EM, Pessoa ALS, Figueiredo TC, Lima LBP. Mental Health of Parents of Children with Congenital Zika Virus Syndrome in Brazil. J Child Fam Stud 2018; 27(4):1207-1215.

38. Catalano D, Holloway L, Mpofu E. Mental health interventions for parent carers of children with autistic spectrum disorder: Practice guidelines from a critical interpretive synthesis (CIS) systematic review. Int J Environ Res Public Health 2018; 15(2):341.

39. Wang Y, Huang Z, Kong F. Parenting stress and life satisfaction in mothers of children with cerebral palsy: The mediating effect of social support. J Health Psychol 2020; 25(3):416-425.

40. Seymour M, Wood C, Giallo R, Jellett R. Fatigue, stress and coping in mothers of children with an autism spectrum disorder. J Autism Dev Disord 2013; 43(7):1547-1554.
41. Dicke T, Marsh HW, Parker PD, Kunter M, Schmeck A, Leutner D. Self-efficacy in classroom management, classroom disturbances, and emotional exhaustion: A moderated mediation analysis of teacher candidates. $J$ Educ Psychol 2014; 106(2):569-583.

42. Hastings RP, Brown T. Behavior Problems of Children With Autism, Parental Self-Efficacy, and Mental Health. Am J Ment Retard 2002; 107(3):222-232.

43. Weiss JA, Tint A, Paquette-Smith M, Lunsky Y. Perceived self-efficacy in parents of adolescents and adults with autism spectrum disorder. Autism 2015; 20(4):425-434.

44. Liyana Amin NA, Tam WWS, Shorey S. Enhancing first-time parents' self-efficacy: A systematic review and meta-analysis of universal parent education interventions' efficacy. Int J Nurs Stud 2018; 82:149-162.

45. Dabrowska A, Pisula E. Parenting stress and coping styles in mothers and fathers of pre-school children with autism and Down syndrome. J Intellect Disabil Res 2010; 54(3):266-280.

Artigo apresentado em 28/01/2019

Aprovado em 12/04/2019

Versão final apresentada em 14/04/2019

Editores chefes: Romeu Gomes, Antônio Augusto Moura da Silva 